\title{
\& A Comparative Study between Four Different Hybrid Local Races of Honey Bee in Some Biological Activity
}

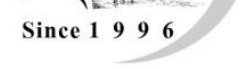

\author{
Ahmed K. Mourad ${ }^{1}$; H.A. Mesbah ${ }^{1}$; Rehab El Assar ${ }^{2}$ and K. Abd \\ ElHamid $^{2}$
}

1.Plant Protection Dept, Faculty of Agriculture Saba Basha, Alexandria University, P.O. Box

21531, Bolkley, Alexandria , Egypt

2.Agriculture Research Centre El Sabaheia ;Alexandria

$$
\text { DOI: } 10.21608 / \text { jalexu.2021.179268 }
$$

\section{Article Information}

Received:June $2^{\text {nd }} 2021$

Revised: June $3^{\text {rd }} 2021$

Accepted:June $16^{\text {th }} 2021$

Published: June $20^{\text {th }} 2021$

ABSTRACT: In this study, experiments were carried out during two seasons of 2017, 2018 using twenty colonies which contain four groups of hybrid strains, each strain contains five replicates. The experiments were carried out on four different hybrid local races bees of, Italian hybrid, Carniolan hybrid and Egyptian hybrid from two different sources (Upper Egypt and Alexandria). This study was established at the experimental apiary of the El-Sabaheia Research Station, Alexandria (27.2831North $\left({ }^{\circ} \mathrm{N}\right)$ and 30.7632 East $^{\circ}(\mathrm{E})$.

Honey and pollen grains were measured during the two seasons 2017 \& 2018 . The honey stored in cells of wax combs was measured by using a typical langstroth frame which is sub-divided into square inches by means of wire for twenty colonies during the two seasons 2017and 2018 to denote the total amount of honey in wax cells per season.

The obtained data showed that the highest mean of honey yield during the season of 2017 was hybrid Carniolan local 1040.85 (inch $^{2}$ ) /colony followed by Egyptian Upper Egypt 1013.65 (inch $\left.{ }^{2}\right)$, then hybrid Italian local

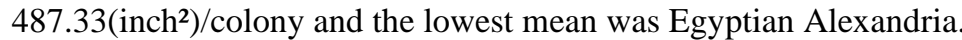

Meanwhile in the season of 2018 the data showed that the highest mean of honey yield was for the Egyptian Upper Egypt 418.88(inch ${ }^{2}$ ) followed by hybrid Italian local $367.84\left(\right.$ inch $\left.^{2}\right)$ then Egyptian Alexandria $303.16\left(\mathrm{inch}^{2}\right)$ and the lowest mean of honey yield was recorded during the season of 2018 for the hybrid Carniolan local $294.80\left(\right.$ inch $\left.^{2}\right) /$ colony .

The stored pollen in cells of wax combs that has been measured by subdivided langstroth frame in square inches was calculated and the area of stored pollen by workers in the twenty colonies were compared.

The results of the current study showed that the collected pollen grains of the selected races during the season of 2017 recorded the highest mean of pollen grains in Egyptian Alexandria 142.77 (inch ${ }^{2}$ )/colony followed by Egyptian upper Egypt 109.31(inch $\left.{ }^{2}\right) /$ colony then hybrid Carniola local 108.07 $\left(\right.$ inch $\left.^{2}\right)$ /colony and the lowest mean of pollen grains was recorded in hybrid Italian local strain $87.82\left(\right.$ inch $\left.^{2}\right) /$ colony .

The highest general mean of pollen grain during season 2018 was recorded in hybrid Italian local 202.84 followed by Egyptian upper Egypt 221.32 then hybrid Carniola local $190.09\left(\right.$ inch $\left.^{2}\right) /$ colony and the lowest general mean was recorded in Egyptian Alexandria 183.04 (inch ${ }^{2}$ )/colony .

Keywords: Pollen grains; Honey; Langstroth frame Colonies

\section{INTRODUCTION}

Honeybee colonies are essential for agriculture and the environment, they ensure plant reproduction by pollination, whilst beekeeping contributes to the development of rural areas. Pollen is the powder found in the flowers. This powder is attached to the hair in the body of the bee and there are pockets in the back legs of the bee called the pollen basket. The bee collects this powder in its body and brings it to the hive. The hive also has traps for pollen grains in which the latter collects and is extracted from the hive. It is considered an ideal food, as it contains 18 vitamins, more than 25 minerals and thirty percent of amino acids and the same amount of sugars. Bees collect nectar, transform it by combining with specific substances of their own deposit, dehydrate, store and leave the honey in the combs to be ripened and matured according to the international standards of honey products . The amount of pollen and brood in the colony can be used to expect the honey yield produced at the end of the season. Nabros (2000); Hegazi et al. (2001) and Taha (2005). 
It is well known that there are three seasons of honey yield in many parts of Egypt according to blooming of plant species that can be available for bees to collect their food (nectar and pollen). For example, citrus honey (first yield of the season) is produced by honey bee workers form the nectar of flowers of different citrus trees at the beginning of spring until the end of flowering period of citrus species. Citrus honey has a light yellowish or golden color with light consistency, light acidic taste and aroma of citrus flavour. Clover honey (second yield) is being produced by honey bee workers throughout the period of May-June during the flowering of clover. Cotton (Gossypium barbadense) honey is also produced at the mid of August (as third yield). Elaidy Waleed et $\boldsymbol{a l}$. (2017).

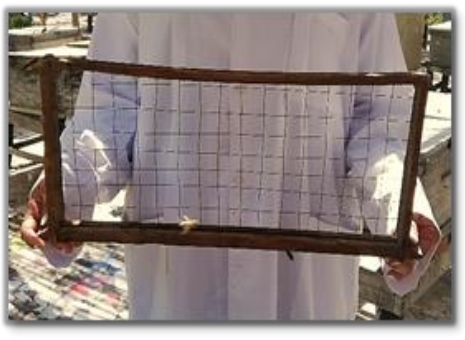

Fig.(1) Measuring Inch square

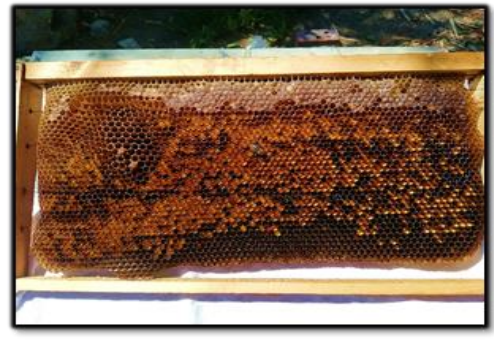

Fig. (2) pollen grains
Therefore, the present work was initiated to study pollen\& Honey gathering activity of the honeybee colonies (four races) under the prevailing local environmental conditions of El-Sabaheia. Region of 2017 till 2018.

\section{MATERIALS AND METHODS \\ Honey stored yield:}

Honey and pollen grains stored were measured during two seasons of 2017, 2018.The honey stored in cells of wax frames was measured by using a typical langstroth frame which is subdivided into square inches by means of wire (AlTikrity et al., 1971 and Hassona, 2006) Fig (1) for twenty colonies during two seasons of 2017, 2018 to denote the total amount of honey in wax cells per season Figs. (2\&3)

\section{Evaluation of stored pollen grains :}

The stored pollen in cells of wax frames that has been measured by subdivided langstroth frame in square inches was calculated and the area of stored pollen by workers in the twenty colonies were compared Fig (4).

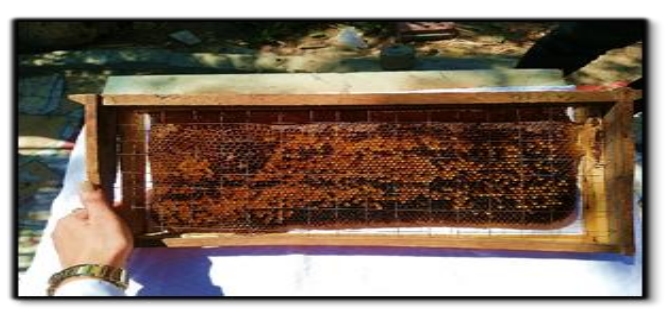

Fig. (4) Measuring pollen by inch square

\section{Statistical analysis}

Using "F" and "L.S.D." tests were performed for the comparison and evaluation of the tested materials. Data were subjected to the analysis of variance test (ANOVA) with mean separation at $5 \%$ levels of significance. Computer programs IRRISTAT and Duncan's Multiple Range test were used to compare the averages of detected results according to the method of Snedecor and Cochran (1967).

\section{RESULTS AND DISCUSSION}

Biological activities of honey bee colonies during seasons 2017 and 2018

Pollen grains measured during season 2017 and $\underline{2018}$

The collected pollen grains of the selected races were measured during season 2017. Table (1) showed that the highest mean of pollen grain was recorded in Egyptian Alexandria 142.77 ( inch $^{2}$ ) followed by Egyptian upper Egypt 109.31(inch ${ }^{2}$ ) then hybrid Carniolan local 108.07 (inch ${ }^{2}$ ) and the lowest mean of pollen grains was recorded in hybrid Italian local strain $87.82\left(\right.$ inch $\left.^{2}\right) /$ colony .

Table (2) showed that the highest general mean of pollen grain during season 2018 was recorded in hybrid Italian local 202.84 followed by Egyptian upper Egypt 221.32 then hybrid Carniola local 190.08 and the lowest general mean was recorded in Egyptian Alexandria $183.04\left(\right.$ inch $\left.^{2}\right) /$ colony .

It could be revealed from these cited results that the highest detected quantities of collected pollen grains in the following months of June and July, was in parallel with the higher estimated areas of sealed brood or/ and honey production .

These results are close to those reported by Bobrzeeki and Wild (1991) who found that the brood area was reduced in colonies of small amount of pollen grain 
On the other hand, Ghazala and Nowar (2014) indicated that mean amounts of pollen trapped during citrus season were $247.66 \mathrm{~g} /$ colony. They also concluded that the mean amount of pollen trapped was 355.00 and $1043.33 \mathrm{~g} /$ colony during the whole season of clover and corn seasons.

Honey yield measured during seasons 2017 and 2018

Honey is produced by honey bees from the nectar of plants, secretions of living parts of plants and excretions of plant sucking insects (honey dew). Bees collect nectar, transform it by combining with specific substances of their own deposit, dehydrate, store and leave the honey in the combs to be ripened and matured according to the international standards of honey products . Sugden and Furgala (1982) stated that the variation of honey production between colonies in the same apiary might be due to variation of genetic makeup of the colony. Kathy and Hultgren (1985) indicated that Carniolin bees can be considered as a good honey production insect which have a good reputation as honey gatherers and they are very prolific. It is well known that there are three seasons of honey yield in many parts of Egypt according to blooming of plant species that can be available for bees to collect their food (nectar and pollen). The apiary of El-Sabahia Research Station in Alexandria, the main flowering season occurs from September to October or November, all the hives produce considerable amounts of honey and pollen grains are collected from Schinus terebinthifolia in October and the average honey yield is about $6.85 \mathrm{~kg}$ per colony

Table (3) showed that the highest mean of honey yield during the season of 2017 was hybrid Carniolan local 1040.85 (inch ${ }^{2}$ )/colony followed by Egyptian Upper Egypt 1013.65 (inch $\left.{ }^{2}\right) /$ colony , then hybrid Italian local 487.33(inch ${ }^{2}$ ) and the lowest mean was Egyptian Alexandria $453.33\left(\mathrm{inch}^{2}\right) /$ colony. These results are in accordance with that reported by the above mentioned authors.

Table (4) showed that the highest mean of honey yield during the season of 2018 was Egyptian Upper Egypt $418.88\left(\mathrm{inch}^{2}\right)$ /colony followed by hybrid Italian local 367.84 (inch $^{2}$ ) then Egyptian Alexandria 303.16 (inch $^{2}$ ) /colony and the lowest mean of honey yield during season 2018 was hybrid Carniola local $294.80\left(\right.$ inch $\left.^{2}\right) /$ colony 
(JAAR) Volume: 26 (3)

Table (1): Comparison between the four races in collecting pollen grains in the season of 2017

\begin{tabular}{|c|c|c|c|c|c|c|c|c|c|c|c|c|c|c|c|c|c|c|c|c|}
\hline \multirow{3}{*}{ Date } & \multicolumn{20}{|c|}{ Strains } \\
\hline & \multicolumn{5}{|c|}{ Hybrid carniola local } & \multicolumn{5}{|c|}{ Hybrid Italian local } & \multicolumn{5}{|c|}{ Egyptian (upper Egypt) } & \multicolumn{5}{|c|}{ Egyptian (Alexandria) } \\
\hline & $1^{*}$ & 2 & 3 & 4 & 5 & 1 & 2 & 3 & 4 & 5 & 1 & 2 & 3 & 4 & 5 & 1 & 2 & 3 & 4 & 5 \\
\hline 23-Mar & 102.00 & 68.00 & 136.00 & 68.00 & 68.00 & 102.00 & 170.00 & 138.00 & 45.00 & 68.00 & 102.00 & 204.00 & 138.00 & 102.00 & 136.00 & 104.00 & 272.00 & 68.00 & 68.00 & 102.00 \\
\hline 6-Apr & 238.00 & 45.00 & 45.00 & 68.00 & 102.00 & 204.00 & 17.00 & 102.00 & 136.00 & 15.00 & 272.00 & 408.00 & 272.00 & 272.00 & 102.00 & 136.00 & 272.00 & 102.00 & 102.00 & 136.00 \\
\hline 20-Apr & 102.00 & 45.00 & 102.00 & 136.00 & 136.00 & 204.00 & 34.00 & 34.00 & 68.00 & 68.00 & 102.00 & 136.00 & 272.00 & 204.00 & 68.00 & 272.00 & 136.00 & 204.00 & 272.00 & 102.00 \\
\hline 4-May & 17.00 & 4.00 & 68.00 & 45.00 & 34.00 & 102.00 & 34.00 & 34.00 & 102.00 & 68.00 & 102.00 & 204.00 & 45.00 & 136.00 & 68.00 & 204.00 & 340.00 & 136.00 & 204.00 & 238.00 \\
\hline 18-May & 136.00 & 170.00 & 68.00 & 204.00 & 204.00 & 34.00 & 5.00 & 45.00 & 170.00 & 102.00 & 68.00 & 204.00 & 272.00 & 68.00 & 170.00 & 136.00 & 136.00 & 68.00 & 408.00 & 340.00 \\
\hline 25-May & 102.00 & 45.00 & 102.00 & 34.00 & 238.00 & 136.00 & 34.00 & 136.00 & 68.00 & 102.00 & 102.00 & 170.00 & 136.00 & 45.00 & 136.00 & 68.00 & 204.00 & 340.00 & 120.00 & 120.00 \\
\hline 15-Jun & 136.00 & 238.00 & 68.00 & 17.00 & 272.00 & 14.00 & 5.00 & 68.00 & 68.00 & 102.00 & 68.00 & 45.00 & 55.00 & 68.00 & 102.00 & 102.00 & 68.00 & 272.00 & 68.00 & 102.00 \\
\hline 29-Jun & 102.00 & 136.00 & 102.00 & 17.00 & 14.00 & 34.00 & 6.00 & 68.00 & 102.00 & 68.00 & 15.00 & 2.00 & 68.00 & 170.00 & 170.00 & 102.00 & 204.00 & 68.00 & 102.00 & 102.00 \\
\hline 13-Jul & 68.00 & 204.00 & 68.00 & 34.00 & 204.00 & 4.00 & 5.00 & 102.00 & 204.00 & 17.00 & 45.00 & 23.00 & 4.00 & 136.00 & 136.00 & 204.00 & 68.00 & 170.00 & 102.00 & 170.00 \\
\hline 27-Jul & 68.00 & 102.00 & 102.00 & 204.00 & 204.00 & 17.00 & 34.00 & 1.00 & 68.00 & 17.00 & 23.00 & 25.00 & 1.00 & 34.00 & 45.00 & 204.00 & 34.00 & 136.00 & 204.00 & 272.00 \\
\hline 10-Aug & 136.00 & 102.00 & 272.00 & 136.00 & 204.00 & 17.00 & 136.00 & 204.00 & 238.00 & 204.00 & 45.00 & 23.00 & 15.00 & 136.00 & 136.00 & 17.00 & 204.00 & 34.00 & 204.00 & 204.00 \\
\hline 24-Aug & 102.00 & 170.00 & 136.00 & 45.00 & 136.00 & 68.00 & 204.00 & 340.00 & 4.00 & 272.00 & 6.00 & 68.00 & 0.50 & 68.00 & 68.00 & 34.00 & 4.00 & 136.00 & 17.00 & 68.00 \\
\hline 7-Sep & 204.00 & 272.00 & 68.00 & 170.00 & 136.00 & 34.00 & 136.00 & 68.00 & 408.00 & 408.00 & 3.00 & 45.00 & 3.00 & 408.00 & 204.00 & 1.00 & 5.00 & 68.00 & 34.00 & 68.00 \\
\hline 21-Sep & 136.00 & 204.00 & 68.00 & 4.00 & 1.00 & 15.00 & 0.50 & 3.00 & 68.00 & 68.00 & 2.00 & 2.00 & 68.00 & 408.00 & 340.00 & 68.00 & 102.00 & 408.00 & 1.00 & 68.00 \\
\hline 5-Oct & 102.00 & 2.00 & 2.00 & 3.00 & 2.00 & 34.00 & 6.00 & 45.00 & 2.00 & 23.00 & 2.00 & 23.00 & 68.00 & 102.00 & 4.00 & 17.00 & 204.00 & 408.00 & 68.00 & 102.00 \\
\hline Mean & 116.73 & 120.47 & 93.80 & 79.00 & 130.33 & 67.93 & 55.10 & 92.53 & 116.73 & 106.80 & 63.80 & 105.47 & 94.50 & 157.13 & 125.67 & 111.27 & 150.20 & 174.53 & 131.60 & 146.27 \\
\hline General mean & \multicolumn{5}{|c|}{$108.07 \mathrm{~b}$} & \multicolumn{5}{|c|}{$87.82 \mathrm{c}$} & \multicolumn{5}{|c|}{$109.31 \mathrm{~b}$} & \multicolumn{5}{|c|}{$142.77 \mathrm{a}$} \\
\hline
\end{tabular}

LSD at $0.05=31.34$ 
(JAAR) Volume: 26 (3)

Table (2): Comparison between the four races in collecting pollen grains in the season of 2018

\begin{tabular}{|c|c|c|c|c|c|c|c|c|c|c|c|c|c|c|c|c|c|c|c|c|}
\hline \multirow{3}{*}{ Date } & \multicolumn{20}{|c|}{ Strains } \\
\hline & \multicolumn{5}{|c|}{ Hybrid camiola local } & \multicolumn{5}{|c|}{ Hybrid Italian local } & \multicolumn{5}{|c|}{ Egyptian (upper Egypt) } & \multicolumn{5}{|c|}{ Egyptian (Alexandria) } \\
\hline & $1^{*}$ & 2 & 3 & 4 & 5 & 1 & 2 & 3 & 4 & 5 & 1 & 2 & 3 & 4 & 5 & 1 & 2 & 3 & 4 & 5 \\
\hline 25-Mar & 66.00 & 66.00 & 66.00 & 33.00 & 132.00 & 33.00 & 66.00 & 132.00 & 264.00 & 132.00 & 33.00 & 264.00 & 297.00 & 33.00 & 165.00 & 132.00 & 132.00 & 132.00 & 132.00 & 33.00 \\
\hline 8-Apr & 264.00 & 66.00 & 297.00 & 165.00 & 99.00 & 198.00 & 264.00 & 264.00 & 264.00 & 198.00 & 198.00 & 99.00 & 297.00 & 165.00 & 231.00 & 165.00 & 66.00 & 66.00 & 297.00 & 99.00 \\
\hline 25-Apr & 330.00 & 132.00 & 330.00 & 132.00 & 264.00 & 264.00 & 198.00 & 198.00 & 132.00 & 264.00 & 132.00 & 132.00 & 264.00 & 198.00 & 264.00 & 198.00 & 132.00 & 132.00 & 330.00 & 132.00 \\
\hline 20-May & 198.00 & 198.00 & 330.00 & 264.00 & 198.00 & 198.00 & 264.00 & 198.00 & 198.00 & 198.00 & 330.00 & 330.00 & 198.00 & 330.00 & 330.00 & 264.00 & 198.00 & 198.00 & 264.00 & 330.00 \\
\hline 3-Jun & 462.00 & 198.00 & 264.00 & 66.00 & 264.00 & 396.00 & 297.00 & 396.00 & 264.00 & 264.00 & 660.00 & 660.00 & 132.00 & 396.00 & 264.00 & 198.00 & 198.00 & 198.00 & 198.00 & 198.00 \\
\hline 17-Jun & 396.00 & 264.00 & 330.00 & 132.00 & 264.00 & 330.00 & 330.00 & 396.00 & 198.00 & 264.00 & 297.00 & 396.00 & 330.00 & 264.00 & 198.00 & 462.00 & 330.00 & 264.00 & 264.00 & 132.00 \\
\hline 1-Jul & 132.00 & 132.00 & 198.00 & 198.00 & 132.00 & 66.00 & 33.00 & 66.00 & 99.00 & 66.00 & 132.00 & 231.00 & 99.00 & 66.00 & 165.00 & 165.00 & 132.00 & 165.00 & 165.00 & 33.00 \\
\hline 15-Jul & 66.00 & 66.00 & 33.00 & 66.00 & 66.00 & 99.00 & 66.00 & 99.00 & 132.00 & 99.00 & 99.00 & 165.00 & 132.00 & 99.00 & 165.00 & 132.00 & 165.00 & 132.00 & 132.00 & 66.00 \\
\hline 12-Aug & 264.00 & 165.00 & 132.00 & 165.00 & 297.00 & 165.00 & 231.00 & 297.00 & 198.00 & 297.00 & 198.00 & 231.00 & 165.00 & 231.00 & 330.00 & 165.00 & 132.00 & 165.00 & 132.00 & 264.00 \\
\hline 26-Aug & 198.00 & 198.00 & 231.00 & 132.00 & 165.00 & 165.00 & 132.00 & 165.00 & 198.00 & 198.00 & 132.00 & 132.00 & 99.00 & 330.00 & 231.00 & 198.00 & 198.00 & 165.00 & 132.00 & 198.00 \\
\hline 9-Sep & 330.00 & 264.00 & 396.00 & 66.00 & 297.00 & 330.00 & 198.00 & 330.00 & 396.00 & 396.00 & 264.00 & 132.00 & 66.00 & 462.00 & 396.00 & 264.00 & 264.00 & 132.00 & 198.00 & 264.00 \\
\hline 23-Sep & 132.00 & 132.00 & 264.00 & 264.00 & 330.00 & 198.00 & 264.00 & 132.00 & 264.00 & 66.00 & 66.00 & 264.00 & 165.00 & 66.00 & 396.00 & 330.00 & 198.00 & 330.00 & 99.00 & 297.00 \\
\hline 7-Oct & 198.00 & 198.00 & 132.00 & 66.00 & 132.00 & 231.00 & 132.00 & 198.00 & 264.00 & 66.00 & 132.00 & 330.00 & 132.00 & 132.00 & 264.00 & 330.00 & 33.00 & 198.00 & 33.00 & 99.00 \\
\hline Mean & 233.20 & 151.80 & 224.40 & 138.60 & 202.40 & 193.60 & 200.20 & 217.80 & 209.00 & 193.60 & 204.60 & 253.00 & 176.00 & 215.60 & 257.40 & 222.20 & 160.60 & 169.40 & 187.00 & 176.00 \\
\hline General mean & \multicolumn{5}{|c|}{$190.08 \mathrm{bc}$} & \multicolumn{5}{|c|}{$202.84 \mathrm{~b}$} & \multicolumn{5}{|c|}{$221.32 \mathrm{a}$} & \multicolumn{5}{|c|}{$183.04 \mathrm{c}$} \\
\hline
\end{tabular}

LSD at $0.05=35.25$ 
(JAAR) Volume: 26 (3)

Table (3): The measured Honey yield of the es in the season of 2017

\begin{tabular}{|c|c|c|c|c|c|c|c|c|c|c|c|c|c|c|c|c|c|c|c|c|}
\hline \multirow{3}{*}{ Date } & \multicolumn{20}{|c|}{ Strains } \\
\hline & \multicolumn{5}{|c|}{ Hybrid carniola local } & \multicolumn{5}{|c|}{ Hybrid Italian local } & \multicolumn{5}{|c|}{ Egyptian (upper Egypt) } & \multicolumn{5}{|c|}{ Egyptian (Alexandria) } \\
\hline & $1^{*}$ & 2 & 3 & 4 & 5 & 1 & 2 & 3 & 4 & 5 & 1 & 2 & 3 & 4 & 5 & 1 & 2 & 3 & 4 & 5 \\
\hline 23-Mar & 884.00 & 952.00 & 204.00 & 1224.00 & 1632.00 & 204.00 & 272.00 & 204.00 & 884.00 & 340.00 & 272.00 & 408.00 & 136.00 & 544.00 & 408.00 & 340.00 & 272.00 & 340.00 & 272.00 & 272.00 \\
\hline 6-Apr & 884.00 & 1496.00 & 204.00 & 2176.00 & 1564.00 & 340.00 & 340.00 & 272.00 & 680.00 & 204.00 & 884.00 & 952.00 & 204.00 & 1224.00 & 1632.00 & 204.00 & 272.00 & 204.00 & 884.00 & 340.00 \\
\hline 20-Apr & 680.00 & 952.00 & 136.00 & 952.00 & 816.00 & 272.00 & 340.00 & 272.00 & 306.00 & 340.00 & 884.00 & 1496.00 & 204.00 & 2176.00 & 1564.00 & 340.00 & 340.00 & 272.00 & 680.00 & 204.00 \\
\hline 4-May & 272.00 & 408.00 & 136.00 & 544.00 & 408.00 & 340.00 & 272.00 & 340.00 & 272.00 & 272.00 & 1088.00 & 952.00 & 170.00 & 1156.00 & 1360.00 & 170.00 & 204.00 & 408.00 & 340.00 & 204.00 \\
\hline 18-May & 408.00 & 408.00 & 170.00 & 544.00 & 544.00 & 136.00 & 68.00 & 408.00 & 340.00 & 136.00 & 952.00 & 136.00 & 952.00 & 816.00 & 272.00 & 340.00 & 272.00 & 306.00 & 340.00 & 272.00 \\
\hline 25-May & 1088.00 & 816.00 & 306.00 & 952.00 & 1224.00 & 272.00 & 374.00 & 544.00 & 816.00 & 272.00 & 408.00 & 408.00 & 170.00 & 544.00 & 544.00 & 136.00 & 68.00 & 408.00 & 340.00 & 136.00 \\
\hline 15-Jun & 1088.00 & 952.00 & 170.00 & \begin{tabular}{|l|}
1156.00 \\
\end{tabular} & 1360.00 & 170.00 & 204.00 & 408.00 & 340.00 & 204.00 & 1088.00 & 816.00 & 306.00 & 952.00 & 1224.00 & 272.00 & 374.00 & 544.00 & 816.00 & 272.00 \\
\hline 29-Jun & 408.00 & 170.00 & 544.00 & 544.00 & 136.00 & 68.00 & 408.00 & 340.00 & 136.00 & 170.00 & 680.00 & 952.00 & 136.00 & 952.00 & 816.00 & 272.00 & 340.00 & 272.00 & 306.00 & 340.00 \\
\hline 13-Jul & 884.00 & 952.00 & 204.00 & 1224.00 & 1632.00 & 204.00 & 272.00 & 204.00 & 884.00 & 340.00 & 1428.00 & 1768.00 & 340.00 & 2312.00 & 1088.00 & 68.00 & 408.00 & 544.00 & 408.00 & 204.00 \\
\hline 27-Jul & 884.00 & 1496.00 & 204.00 & 2176.00 & 1564.00 & 340.00 & 340.00 & 272.00 & 680.00 & 204.00 & 408.00 & 408.00 & 170.00 & 544.00 & \begin{tabular}{|l|}
544.00 \\
\end{tabular} & 136.00 & 68.00 & 408.00 & 340.00 & 136.00 \\
\hline 10-Aug & 680.00 & 952.00 & 136.00 & 952.00 & 816.00 & 272.00 & 340.00 & 272.00 & 306.00 & 340.00 & 1632.00 & 1904.00 & 1088.00 & 1632.00 & 1224.00 & 408.00 & 1292.00 & 1088.00 & 816.00 & 748.00 \\
\hline 24-Aug & 1428.00 & 1768.00 & 340.00 & 2312.00 & 1088.00 & 68.00 & 340.00 & 408.00 & 408.00 & 204.00 & 1360.00 & 2108.00 & 544.00 & 1632.00 & 1564.00 & 476.00 & 816.00 & 340.00 & 476.00 & 1768.00 \\
\hline 7-Sep & 2040.00 & \begin{tabular}{|l|}
1904.00 \\
\end{tabular} & 2448.00 & 2176.00 & 1224.00 & 1224.00 & 1496.00 & 476.00 & 1904.00 & 816.00 & 1632.00 & 1904.00 & 1088.00 & 1632.00 & 1224.00 & 408.00 & 1292.00 & 1088.00 & 816.00 & 748.00 \\
\hline 21-Sep & 1360.00 & 2108.00 & 544.00 & 1632.00 & \begin{tabular}{|l|}
1564.00 \\
\end{tabular} & 476.00 & 816.00 & 1632.00 & 748.00 & 1088.00 & 1428.00 & 1768.00 & 340.00 & 2312.00 & 1088.00 & 68.00 & \begin{tabular}{|l|}
408.00 \\
\end{tabular} & \begin{tabular}{|l|}
408.00 \\
\end{tabular} & 408.00 & 204.00 \\
\hline $5-\mathrm{Oct}$ & 1292.00 & 2040.00 & 1904.00 & 2448.00 & 2176.00 & 1496.00 & 1224.00 & 1496.00 & 476.00 & 1904.00 & 1632.00 & 1632.00 & 1088.00 & 1496.00 & 1224.00 & 408.00 & 1292.00 & 952.00 & 816.00 & 748.00 \\
\hline Mean & 952.00 & 1158.27 & 510.00 & 1400.80 & 1183.20 & 392.13 & 473.73 & 503.20 & 612.00 & 455.60 & 1051.73 & 1174.13 & 462.40 & 1328.27 & 1051.73 & 269.73 & 514.53 & 505.47 & 537.20 & 439.73 \\
\hline General mean & \multicolumn{5}{|c|}{$1040.85 \mathrm{a}$} & \multicolumn{5}{|c|}{$487.33 \mathrm{~b}$} & \multicolumn{5}{|c|}{$1013.65 \mathrm{a}$} & \multicolumn{5}{|c|}{$453.33 \mathrm{~b}$} \\
\hline
\end{tabular}

LSD 0.05 $=149.045$ 
(JAAR) Volume: 26 (3)

Table (4): The measured Honey yield of the four races in the season of 2018

\begin{tabular}{|c|c|c|c|c|c|c|c|c|c|c|c|c|c|c|c|c|c|c|c|c|}
\hline \multirow{3}{*}{ Date } & \multicolumn{20}{|c|}{ Strains } \\
\hline & \multicolumn{5}{|c|}{ Hybrid camiola local } & \multicolumn{5}{|c|}{ Hybrid Italian local } & \multicolumn{5}{|c|}{ Egyptian (upper Egypt) } & \multicolumn{5}{|c|}{ Egyptian (Alexandria) } \\
\hline & $1 *$ & 2 & 3 & 4 & 5 & 1 & 2 & 3 & 4 & 5 & 1 & 2 & 3 & 4 & 5 & 1 & 2 & 3 & 4 & 5 \\
\hline 25-Mar & 66.00 & 132.00 & 132.00 & 66.00 & 264.00 & 132.00 & 528.00 & 396.00 & 528.00 & 396.00 & 528.00 & 396.00 & 462.00 & 396.00 & 462.00 & 198.00 & 264.00 & 132.00 & 198.00 & 0.00 \\
\hline 8-Apr & 132.00 & 66.00 & 264.00 & 132.00 & 66.00 & 99.00 & 132.00 & 396.00 & 330.00 & 297.00 & 396.00 & 198.00 & 528.00 & 66.00 & 330.00 & 330.00 & 264.00 & 0.00 & 660.00 & 66.00 \\
\hline 25-Apr & 66.00 & 132.00 & 132.00 & 66.00 & 132.00 & 66.00 & 132.00 & 264.00 & 396.00 & 264.00 & 330.00 & 132.00 & 330.00 & 33.00 & 264.00 & 396.00 & 132.00 & 66.00 & 396.00 & 132.00 \\
\hline 6-May & 132.00 & 132.00 & 396.00 & 264.00 & 132.00 & 132.00 & 132.00 & 462.00 & 396.00 & 396.00 & 330.00 & 462.00 & 264.00 & 396.00 & 198.00 & 264.00 & 264.00 & 132.00 & 396.00 & 132.00 \\
\hline 20-May & 198.00 & 198.00 & 330.00 & 330.00 & 198.00 & 198.00 & 198.00 & 396.00 & 330.00 & 330.00 & 396.00 & 396.00 & 330.00 & 330.00 & 264.00 & 198.00 & 198.00 & 198.00 & 330.00 & 198.00 \\
\hline 3-Jun & 264.00 & 264.00 & 264.00 & 132.00 & 396.00 & 528.00 & 396.00 & 429.00 & 264.00 & 330.00 & 528.00 & 462.00 & 264.00 & 594.00 & 462.00 & 264.00 & 264.00 & 330.00 & 396.00 & 264.00 \\
\hline 17-Jun & 396.00 & 396.00 & 330.00 & 264.00 & 528.00 & 396.00 & 528.00 & 462.00 & 396.00 & 396.00 & 396.00 & 660.00 & 528.00 & 330.00 & 528.00 & 528.00 & 528.00 & 396.00 & 462.00 & 396.00 \\
\hline 1-Jul & 66.00 & 33.00 & 132.00 & 66.00 & 66.00 & 132.00 & 66.00 & 132.00 & 132.00 & 132.00 & 132.00 & 198.00 & 132.00 & 198.00 & 66.00 & 99.00 & 132.00 & 198.00 & 132.00 & 66.00 \\
\hline 15-Jul & 66.00 & 33.00 & 66.00 & 66.00 & 66.00 & 132.00 & 66.00 & 132.00 & 165.00 & 198.00 & 132.00 & 198.00 & 132.00 & 198.00 & 132.00 & 132.00 & 165.00 & 198.00 & 132.00 & 132.00 \\
\hline 29-Jul & 132.00 & 396.00 & 198.00 & 132.00 & 330.00 & 396.00 & 132.00 & 396.00 & 132.00 & 396.00 & 198.00 & 330.00 & 330.00 & 165.00 & 264.00 & 264.00 & 66.00 & 462.00 & 429.00 & 264.00 \\
\hline 12-Aug & 198.00 & 330.00 & 330.00 & 330.00 & 330.00 & 462.00 & 198.00 & 330.00 & 198.00 & 330.00 & 330.00 & 198.00 & 330.00 & 363.00 & 231.00 & 363.00 & 330.00 & 198.00 & 495.00 & 462.00 \\
\hline 26-Aug & 396.00 & 429.00 & 396.00 & 264.00 & 429.00 & 396.00 & 330.00 & 429.00 & 396.00 & 396.00 & 330.00 & 330.00 & 396.00 & 528.00 & 396.00 & 429.00 & 198.00 & 264.00 & 297.00 & 330.00 \\
\hline 9-Sep & 462.00 & 594.00 & 528.00 & 132.00 & 462.00 & 429.00 & 264.00 & 396.00 & 462.00 & 594.00 & 594.00 & 330.00 & 528.00 & 264.00 & 198.00 & 462.00 & 396.00 & 330.00 & 198.00 & 66.00 \\
\hline 23-S ep & 792.00 & 726.00 & 924.00 & 330.00 & 792.00 & 792.00 & 396.00 & 990.00 & 528.00 & 1056.00 & 660.00 & 1254.00 & 924.00 & 924.00 & 594.00 & 726.00 & 858.00 & 594.00 & 792.00 & 462.00 \\
\hline 7-Oct & 726.00 & 726.00 & 858.00 & 462.00 & 924.00 & 924.00 & 264.00 & 1056.00 & 726.00 & 1056.00 & 264.00 & 2112.00 & 1188.00 & 1452.00 & 924.00 & 132.00 & 462.00 & 660.00 & 792.00 & 198.00 \\
\hline Mean & 272.80 & 305.80 & 352.00 & 202.40 & 341.00 & 347.60 & 250.80 & 444.40 & 358.60 & 437.80 & 369.60 & 510.40 & 444.40 & 415.80 & 354.20 & 319.00 & 301.40 & 277.20 & 407.00 & 211.20 \\
\hline General mean & \multicolumn{5}{|c|}{$294.80 \mathrm{c}$} & \multicolumn{5}{|c|}{$367.84 \mathrm{~b}$} & \multicolumn{5}{|c|}{$418.88 \mathrm{a}$} & \multicolumn{5}{|c|}{$303.16 \mathrm{c}$} \\
\hline
\end{tabular}

LSD 0.05 = 107.71 
4.REFERENCES

Al-Tikrity, W. S., A.W Benton, R.C. Hillman and W.W. Jr

Clarke (2015). The relationship between the amount of unsealed brood in honeybee colonies and their pollen collection. J. Apic. Res. 11:9-12.

Bobrzecki ,J. and J. Wild (1991) . The influence of pollen trapping 3 types of pollen trps on the development and productivity of honey bee colonies . Pszczelinczezesz Novkowe, 35:19-27

Elaidy Waleed, K. M. and A.T. Abd El-Monem (2017). Comparative Evaluation of Four Honeybee Races According To Pollen Storage and Worker Brood Rearing Activities under Natural Conditions, Pharmacy and Biological Sciences 12(1) 40-49.

Ghazala, Naglaa A. and E. E. Nowar (2014). Survey of different pollen sources gathering by honey bee at Qunatir Al- khiria, Qaluobia Governorate. J. Plant Prot. Path., Mansoura Univ., 5(6):755-771.

Hassona, Nadia M. (2006). Efficiency of the Italian honey bee queen, Apis mellifera L. (Hymenoptera: Apidae) and its hybrids under the Egyptian environment. M.Sc. Thesis. Fac. Agric. (Saba Basha), Alex. Univ., Egypt

Hegazi, A.G., Moharram, N.Z., Abd-Allah, F.A., Nour, M.S. and Khair, A.M. (2001). Antibacterial activity of different Egyptian honeys in relation to some bee products. Egypt. J. Vet. Sci., 36: 31-42.

Kathy, H. and R. Hultgren (1985). What are all these queens about?. Gl. Bee. Cult., 4: 184- 185.

Nabors, R. (2000). The effects of spring feeding pollen substitute to colonies of Apis mellifera. American Bee Journal 140: 322-323.

Snedecor, G.W. and W.G. Cochran (1967). Statistical Methods. 6th Edn, Oxford and IBH Publishing Co. Pvt. Ltd., New Delhi, India, Pages: 593.

Sugden, M.A. and B. Furgala (1982). Evaluation of six commercial honeybee (Apis mellifera L.) stocks used in Minnesota. A. Bee J., 122 (4): 283- 286.

Taha, E. K. A. (2005). Studies on honeybee (Apis mellifera L.). Ph. D. Thesis, Faculty of Agriculture, Tanta University, 159p. Trends Ecol Evol $14: 312-315$ 


\title{
الملخص العربي
}

\section{دراسة مقارنة بين أربعة سلالات هجين محلية مختلفة لنحل العسل في بعض الانثطة الحيوية}

\author{
أحمد كمال مراد' ,حسن عبدالحميد مصباح',رحاب الأعصر ',خالد عبد الحميد'
}

\author{
1-قسم وقاية النبات-كلية الزراعة ساباباشا-جامعة الاسكندرية \\ r-مركز البحوث الزراعية بالصبحية-الاسكندرية
}

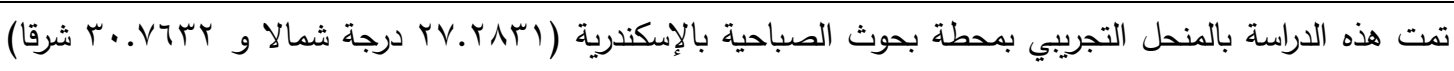

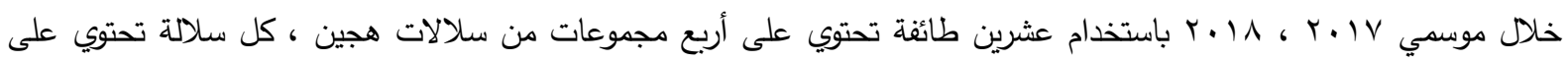

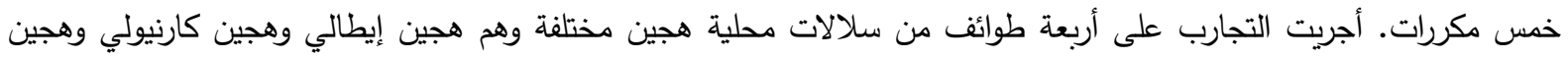

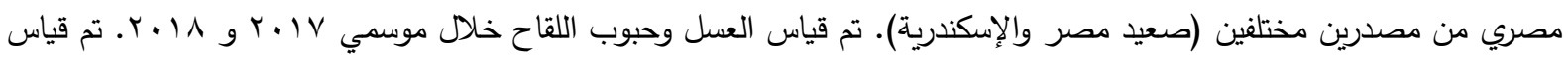

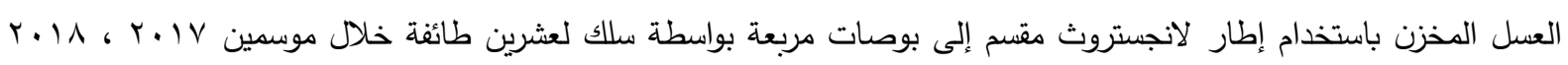

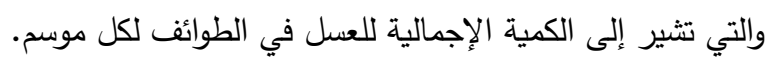

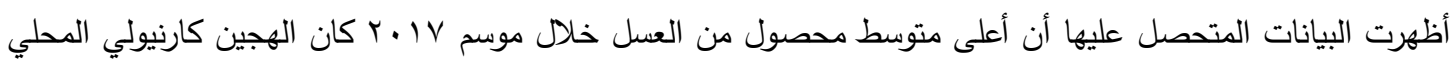

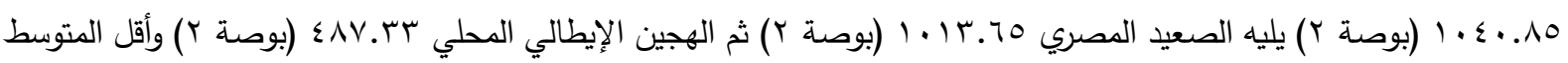

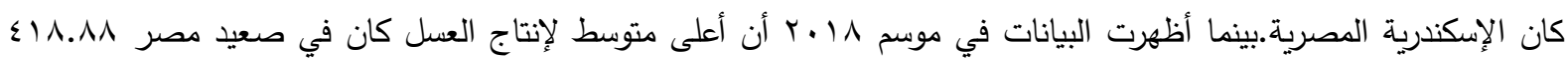

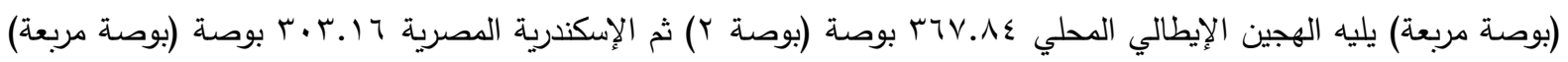

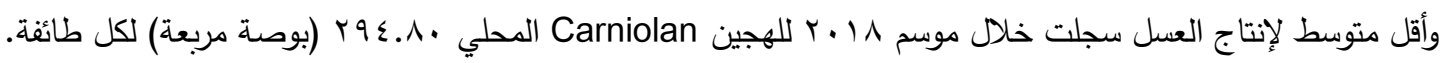
تم حساب حبوب اللقاح المخزنة في الطوائف بواسطة إطار langstroth المقسم بالبوصة المربعة وتمت مقارنة مساحة حبوب اللقاح المخزنة في الطوائف العشرين.

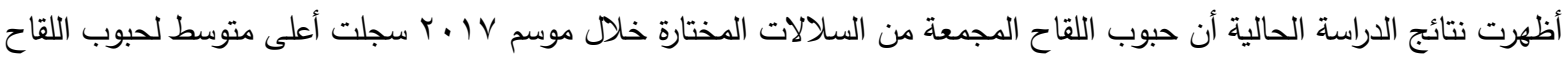

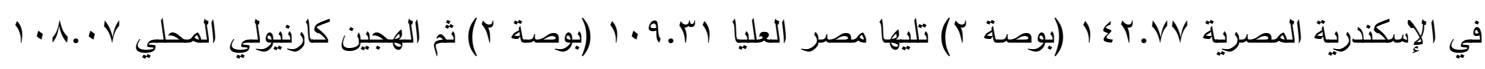
( بوصة2) اطائفة وسجل أدنى متوسط لحبوب اللقاح في السلالة الهجين الإيطالية r.A. 1بوصة مربعة) اطائفة.

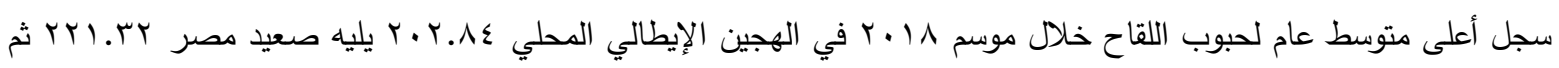

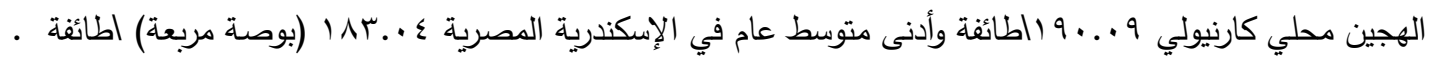
ومن الملاحظ أن الهجين الكرينيولي تنتج كمية كبيرة من العسل المخزن والهجين المصري أعلي كمية من حبوب اللقاح المخزنة بصفة عامة. 